\title{
Isolation Method and
}

\section{Characterization of Outer}

Membranes Vesicles of Helicobacter pylori Grown in a Chemically Defined Medium

\author{
Joana Melo ${ }^{1,2,3}$, Vanessa Pinto 1,2, Tânia Fernandes 1,2, Ana R. Malheiro', Hugo Osório 1,2,4, \\ Ceu Figueiredo ${ }^{1,2,4 * \dagger}$ and Marina Leite ${ }^{1,2,4 * t}$ \\ ${ }^{1}$ i3S - Instituto de Investigação e Inovação em Saúde, Universidade do Porto, Porto, Portugal, ${ }^{2}$ Ipatimup - Instituto \\ de Patologia e Imunologia Molecular da Universidade do Porto, Porto, Portugal, ${ }^{3}$ ICBAS - Instituto de Ciências Biomédicas \\ Abel Salazar, Universidade do Porto, Porto, Portugal, ${ }^{4}$ Departamento de Patologia, Faculdade de Medicina da Universidade \\ do Porto, Porto, Portugal
}

OPEN ACCESS

Edited by:

Christine Josenhans, Ludwig Maximilian University

of Munich, Germany

Reviewed by:

Mario M. D'Elios,

University of Florence, Italy

Stefan Schild,

University of Graz, Austria

${ }^{*}$ Correspondence:

Marina Leite

mleite@ipatimup.pt

Ceu Figueiredo

cfigueiredo@ipatimup.pt

${ }^{\dagger}$ These authors have contributed equally to this work

Specialty section: This article was submitted to

Infectious Diseases,

a section of the journal

Frontiers in Microbiology

Received: 15 January 2021 Accepted: 27 April 2021

Published: 02 June 2021

Citation:

Melo J, Pinto V, Fernandes T, Malheiro AR, Osório H, Figueiredo C and Leite M (2021) Isolation Method and Characterization of Outer Membranes Vesicles of Helicobacter pylori Grown in a Chemically Defined Medium. Front. Microbiol. 12:654193.

doi: 10.3389/fmicb.2021.654193
Outer membrane vesicles (OMVs) are small vesicles constitutively shed by all Gramnegative bacterium, which have been proposed to play a role in Helicobacter pylori persistence and pathogenesis. The methods currently available for the isolation of H. pylori OMVs are diverse and time-consuming, raising the need for a protocol standardization, which was the main aim of this study. Here, we showed that the chemically defined F12 medium, supplemented with cholesterol, nutritionally supports bacterial growth and maintains $H$. pylori viability for at least $72 \mathrm{~h}$. Additionally, we developed an abridged protocol for isolation of OMVs from these bacterial cultures, which comprises a low-speed centrifugation, supernatant filtration through a $0.45 \mu \mathrm{m}$ pore, and two ultracentrifugations for OMVs' recovery and washing. Using this approach, a good yield of highly pure bona fide OMVs was recovered from cultures of different $H$. pylori strains and in different periods of bacterial growth, as assessed by nanoparticle tracking analysis, transmission electron microscopy (TEM), and proteomic analyses, confirming the reliability of the protocol. Analysis of the proteome of OMVs isolated from $\mathrm{H}$. pylori F12-cholesterol cultures at different time points of bacterial growth revealed differentially expressed proteins, including the vacuolating cytotoxin VacA. In conclusion, this work proposes a time- and cost-efficient protocol for the isolation of $\mathrm{H}$. pylori OMVs from a chemically defined culture medium that is suitable for implementation in research and in the biopharmaceutical field.

Keywords: Helicobacter pylori, bacterial outer membrane vesicles, defined growth medium, OMVs isolation method, proteomics

\section{INTRODUCTION}

Helicobacter pylori infects about half of the human population and remains the most common chronic infection in the world (Hooi et al., 2017). This Gram-negative bacterium is acquired in childhood, and unless treated, it establishes a life-long infection of the host gastric mucosa. Persistent $H$. pylori infection is associated with various clinical outcomes, such as peptic ulcer 
disease and gastric cancer, both adenocarcinoma and mucosaassociated lymphoid tissue (MALT) lymphoma (Atherton, 2006; Cover and Blaser, 2009).

H. pylori-mediated diseases, as well as bacterial colonization, survival, and persistence in the gastric mucosa depend upon the concerted action of multiple virulence factors and mechanisms (Blaser and Atherton, 2004). The secretion of outer membrane vesicles (OMVs) emerged as an additional means to facilitate such processes in $H$. pylori infection. It has been shown that $H$. pylori OMVs are internalized and deliver their cargo into epithelial cells, resulting in cellular alterations, such as vacuolization, elongation, modulation of proliferation, and a pro-inflammatory immune response (Chatterjee and Das, 1967; Devoe and Gilchrist, 1973; Hoekstra et al., 1976; Fiocca et al., 1999; Heczko et al., 2000; Ismail et al., 2003; Chitcholtan et al., 2008; Parker et al., 2010). The full contribution of OMVs to bacteria-host and bacteria-bacteria interactions is yet to be disclosed, considering that their effects may extend to locations distant from the locally circumscribed bacterial niche (Kulp and Kuehn, 2010).

Outer membrane vesicles are a type of membrane vesicles (MVs) secreted by virtually all Gram-negative bacteria. They have a spherical structure ranging from 20 to $500 \mathrm{~nm}$ in size, formed through the blebbing of the bacterial outer membrane (OM), enclosing periplasmic and cytoplasmic proteins, genetic material, and lipids (Kulp and Kuehn, 2010). Although there are other types of MVs secreted by different organisms, OMVs are the only type of MVs secreted by $H$. pylori described so far (Deatherage and Cookson, 2012). Even though OMVs are naturally secreted, vesiculation is strongly influenced by the bacterial growth stage and various environmental and stress conditions (Katsui et al., 1982; Thompson et al., 1985; Willen et al., 2000; Olofsson et al., 2010). H. pylori vesiculation, in particular, was shown to increase as bacterial growth progresses from the logarithmic to the stationary phase, and to associate with the morphological transition into the coccoid shape (Willen et al., 2000; Olofsson et al., 2010).

The current methods described for the isolation of $H$. pyloriderived OMVs are diverse and time-consuming. They include several ultracentrifugation and/or density gradient centrifugation steps to purify and recover OMVs, after an initial low-speed centrifugation and culture medium filtration intended to remove the bacteria (Kim et al., 2014; Klimentova and Stulik, 2015). Besides being laborious, the existing methods rely on complex and chemically undefined media for bacterial growth, such as Brucella Broth (BB) or Brain Heart Infusion (BHI), owing to the widely held notion that $H$. pylori is a fastidious organism and requires such enriched media to grow (Keenan et al., 2000; Mullaney et al., 2009; Kaparakis et al., 2010; Zavan et al., 2019). Nonetheless, Testerman et al. have demonstrated that H. pylori has few nutritional requirements and can grow in the chemically defined Ham's-F12 (F12) liquid medium. Furthermore, they have shown that supplementation of F12 with cholesterol, bovine serum albumin, or fetal bovine serum, can further enhance H. pylori growth (Testerman et al., 2001, 2006).

Here, we developed a simpler and faster method to isolate OMVs from $H$. pylori, based on a chemically defined medium for bacterial growth. This enables the standardization of the isolation method, which is pivotal for downstream applications. Accordingly, we used the F12 liquid medium supplemented with cholesterol for bacterial growth, and carried out the isolation of OMVs with one low-speed centrifugation and a $0.45 \mu \mathrm{m}$ filtration to remove bacteria while allowing the recovery of all size-range OMVs, one ultracentrifugation step for vesicle purification, and a last ultracentrifugation as a final wash. Following this approach, a high yield of pure and bona fide spherical $H$. pylori OMVs were recovered.

\section{MATERIALS AND METHODS}

\section{Helicobacter pylori Strains and Growth Conditions in TSA Plates}

Helicobacter pylori strains 26695 (ATCC $^{\circledR}$ 700392, cagA $^{+}$, vacA s1/m1), 60190 (ATCC ${ }^{\circledR} 49503$, cag $^{+}, v a c A s 1 / m 1$ ) and Tx30a (ATCC ${ }^{\circledR} 51932 ; \operatorname{cag}^{-}$, vacA s2/m2) were routinely cultured in Trypticase $^{\mathrm{TM}}$ Soy Agar (TSA) supplemented with 5\% Sheep Blood (Becton, Dickinson and Company, Franklin Lakes, NJ, United States) and incubated in a sealed jar with a microaerophilic atmosphere (GENBox microaer; bioMérieux S.A., Marcy l'Etoile, France) at $37^{\circ} \mathrm{C}$ for $48 \mathrm{~h}$. Bacteria were sub-cultured for a maximum of 12 passages.

\section{Helicobacter pylori Growth in Liquid Cultures}

Helicobacter pylori previously grown in TSA plates for $48 \mathrm{~h}$ was collected with $1 \mathrm{~mL}$ of either sterile Ham's F12 with L-glutamine medium (\#L0135-500; Biowest, Nuaillé, France) supplemented with $1 \times$ cholesterol $\left(\right.$ Gibco $^{\circledR}$, Thermo Fisher Scientific, Waltham, MA, United States) (hereafter designated as F12-cholesterol), or $\mathrm{BBL}^{\mathrm{TM}}$ Brucella broth (BB; \#211088; BD Biosciences, San Jose, CA, United States) supplemented with $5 \%$ fetal bovine serum (FBS; HyClone ${ }^{\mathrm{TM}}$, GE Healthcare Life Sciences, United States) (abbreviated as BB-FBS). The optical density at $600 \mathrm{~nm}$ $\left(\mathrm{OD}_{600}\right)$ was measured using a spectrophotometer (Genesys20; Thermo Fisher Scientific), in polystyrene cuvettes (Thermo Fisher Scientific). The culture medium of the corresponding bacterial culture was used as the blank solution to calibrate the spectrophotometer to $100 \%$ absorbance.

The initial $\mathrm{OD}_{600}$ of the bacterial suspension was adjusted to $0.02\left(\sim 6 \times 10^{6}\right.$ colony forming units $\left.-\mathrm{CFUs} / \mathrm{mL}\right)$ in $200 \mathrm{~mL}$ of F12-cholesterol or BB-FBS. Bacteria were grown in a $500 \mathrm{~mL}$ Schott flask, placed in a sealed jar, under microaerophilic conditions, at $37^{\circ} \mathrm{C}$, and with a constant rotation of $100 \mathrm{rpm}$ (SI600 Large Shaking Incubator; Stuart, Staffordshire, United Kingdom) for the defined periods of time, 24, 48, 64, or $72 \mathrm{~h}$. Bacterial growth was monitored at the referred time points by measuring the $\mathrm{OD}_{600}$ of the liquid bacterial cultures.

\section{Bacterial Viability}

The viability of $H$. pylori grown in liquid media was assessed by two methods: CFUs counting and flow cytometry using the LIVE/DEAD BacLight Bacterial Viability Kit (Thermo Fisher Scientific), according to the manufacturers' instructions. 
For the CFUs counting, at the referred time points, $100 \mu \mathrm{L}$ of bacterial suspension was collected, serially diluted by 10 fold in $\mathrm{BB}$, and $10 \mu \mathrm{L}$ of each dilution was plated on TSA plates, in quadruplicates. Plates were incubated in a sealed jar with a microaerophilic atmosphere, at $37^{\circ} \mathrm{C}$ for $48 \mathrm{~h}$. At this time, colonies were counted and the number of CFUs/mL was calculated (CFUs/mL $=$ number of colonies $\div$ inoculum $\times$ dilution factor $\times 1,000 \mu \mathrm{L}$ ).

For the LIVE/DEAD assay, a bacterial suspension of $2 \times 10^{6}$ cells was filtered through a $10 \mu \mathrm{m}$ CellTrics ${ }^{\circledR}$ filter (Sysmex Partec, Göerlitz, Germany) to remove aggregates. Bacteria were then stained with $0.5 \mathrm{nM}$ SYTO9, a cell-permeant green fluorescent nucleic acid stain that labels both live and dead cells, and with $2 \mu \mathrm{g} / \mathrm{mL}$ propidium iodide, a nucleic acid red-fluorescent dye that labels non-viable cells with damaged membranes, for $15 \mathrm{~min}$ at room temperature (RT) and in the dark. Data acquisition was immediately performed on a FACSCanto II cytometer (BD Biosciences), and analyzed using the FlowJo ${ }^{\mathrm{TM}}$ version 10 software (BD Biosciences). SYTO9 ${ }^{+}$ stained bacterial cells were defined as $\mathrm{SYTO}^{+} \mathrm{PI}^{-}$(live) or SYTO9 $^{+} \mathrm{PI}^{+}$(dead) and results are shown as the frequency of gated cells. SYTO9 and PI single stainings and unstained samples were used as controls.

\section{Isolation of OMVs From Bacterial Liquid Cultures}

Isolation of $H$. pylori-derived OMVs was performed from the supernatant of the liquid bacterial cultures. At the specified time points of bacterial growth, $200 \mathrm{~mL}$ of bacterial cultures were subjected to low-speed centrifugation at $15,000 \times g$, for $15 \mathrm{~min}$ at $4^{\circ} \mathrm{C}$, in a JA25-50 rotor (Avanti J-25; Beckman Coulter, Fullerton, CA, United States), to pellet bacteria. The bacteriafree supernatant was filtered through a $0.45 \mu \mathrm{m}$ cellulose acetate bottle-top filter unit (Corning, NY, United States), and then OMVs were pelleted by ultracentrifugation at $200,000 \times g$, for $90 \mathrm{~min}$ at $4^{\circ} \mathrm{C}$, in a $70 \mathrm{Ti}$ rotor (Optima XE-100; Beckman Coulter). OMVs from the different centrifuge tubes were pooled and washed once in $20 \mathrm{~mL}$ sterile $0.9 \% \mathrm{NaCl}$ solution (saline) (Braun; Kronberg im Taunus, Germany) $(200,000 \times g$, $\left.90 \mathrm{~min}, 4^{\circ} \mathrm{C}\right)$, resuspended in $100 \mu \mathrm{L}$ saline and frozen at $-80^{\circ} \mathrm{C}$ until use.

\section{Transmission Electron Microscopy}

The morphology and purity of $H$. pylori cultures grown in F12-cholesterol and of $H$. pylori-derived OMVs were confirmed by transmission electron microscopy (TEM). The presence of protein aggregates in the liquid media was also evaluated by TEM. For negative staining, $7 \mu \mathrm{L}$ of sample (bacterial suspensions, OMVs or liquid medium) was mounted in Formvar/carbon film-coated mesh nickel grids (Electron Microscopy Sciences, Hatfield, PA, United States), and after the excess liquid was removed, $2 \mu \mathrm{L}$ of aqueous $1 \%$ uranyl acetated solution (\#22400; Electron Microscopy Sciences) were added onto the grids. For the ultrastructure analysis, OMVs samples were fixed in a solution of $2 \%$ glutaraldehyde (\#16316; Electron Microscopy sciences) with $2.5 \%$ formaldehyde (\#15713; Electron Microscopy sciences) in $0.1 \mathrm{M}$ sodium cacodylate buffer ( $\mathrm{pH} 7.4$ ) for $1 \mathrm{~h}$, at RT, and post fixed in 1\% osmium tetroxide (\#19190; Electron Microscopy Sciences) diluted in $0.1 \mathrm{M}$ sodium cacodylate buffer. After ultracentrifugation $\left(200,000 \times g, 90 \mathrm{~min}, 4^{\circ} \mathrm{C}\right)$, the pellet was resuspended in Histogel $^{\mathrm{TM}}$ (HG-4000-012, Thermo Fisher Scientific) and then stained with aqueous $1 \%$ uranyl acetate solution overnight, dehydrated and embedded in Embed812 resin (\#14120; Electron Microscopy sciences). Ultra-thin sections (50 nm thickness) were cut on a RMC Ultramicrotome (PowerTome, United States) using Diatome diamond knifes (DDK, Wilmington, DE, United States), mounted on mesh nickel grids (Electron Microscopy Sciences), and stained with uranyl acetate substitute (\#11000; Electron Microscopy Sciences) and lead citrate (\#11300; Electron Microscopy Sciences) for $5 \mathrm{~min}$ each. Negative staining samples and thin sections were examined under a JEOL JEM 1400 transmission electron microscope (JEOL, Tokyo, Japan) and images were digitally recorded using a CCD digital camera Orius 1100W (Tokyo, Japan).

\section{Scanning Electron Microscopy}

For scanning electron microscopy (SEM) analysis, $5 \mathrm{~mL}$ of bacterial suspension from F12-cholesterol cultures were prefixed in $2.5 \%$ glutaraldehyde solution, diluted in $0.2 \mathrm{M}$ sodium phosphate buffer $\left(0.2 \mathrm{M} \mathrm{Na}_{2} \mathrm{HPO}_{4} \cdot 2 \mathrm{H}_{2} \mathrm{O}\right.$ and $0.2 \mathrm{M} \mathrm{NaH}_{2} \mathrm{PO}_{4}$. $\mathrm{H}_{2} \mathrm{O}$ in $\mathrm{H}_{2} \mathrm{O}$; $\mathrm{PB} ; \mathrm{pH} 7.3$ ), for $24 \mathrm{~h}$ at RT. After two washes in $\mathrm{PB}$, samples were post-fixed in $2 \%$ osmium tetroxide for $48 \mathrm{~h}$ at $\mathrm{RT}$, rinsed in distilled water, and dehydrated in graded ethanol solutions of $50,70,80$, and $95 \%$, with two final $100 \%$ ethanol changes, for $10 \mathrm{~min}$ in each dilution. Samples were chemically dried in Hexamethyldisilazane (HMDS) (Sigma-Aldrich Co., St. Louis, MO, United States) by a first incubation in 50\% HMDS diluted in absolute ethanol for $24 \mathrm{~h}$, followed by a 15 min incubation in $100 \%$ HMDS. Samples were left to airdry in a fume hood at RT. Dried samples were mounted on an aluminum stub with double-sided adhesive carbon tape and sputter-coated with a thin film of $\mathrm{Au} / \mathrm{Pd}$, to improve the electrically conducting properties of the sample surface. Image acquisition was performed using a High resolution (Schottky) Environmental Scanning Electron Microscope with X-Ray Microanalysis and Electron Backscattered Diffraction analysis (FEI Quanta 400 FEG ESEM/EDAX Genesis X4M; Thermo Fisher Scientific).

\section{Nanoparticle Tracking Analysis of OMVs}

The quantification and sizing of the OMVs were determined using a NS300 particle-size tracker with the Nanosight NTA 3.0 software (Malvern, Worcestershire, United Kingdom). Samples were diluted $(1: 40,000)$ in saline to achieve a concentration of $10^{7}-10^{9}$ particles $/ \mathrm{mL}$ for the analysis. Under controlled fluid flow, three measurements (videos of $30 \mathrm{~s}$ each) of each sample were acquired as technical replicates and results were averaged. Reads were performed using the camera level adjusted to a value between 14 and 16, and a detection threshold fixed at five. The sample chamber was flushed with sterile PBS between samples, to avoid cross-contamination. 


\section{Protein Quantification of OMVs}

A total of $10^{11}$ OMVs were diluted in $4 \times$ Laemmli buffer (Bio-Rad Laboratories Inc.) with $\beta$-mercaptoethanol (SigmaAldrich), denaturated at $95^{\circ} \mathrm{C}$ for $5 \mathrm{~min}$ and loaded onto $7.5 \%$ sodium dodecyl sulfate-polyacrylamide gels (SDS-PAGE). After electrophoresis, gels were stained with $25 \mathrm{~mL}$ of BlueSafe (NZYTech, Lisbon, Portugal), for $1 \mathrm{~h}$ at RT with gentle rotation, washed with distilled water for $10 \mathrm{~min}$ three times, and visualized in a GS-800 Calibrated Densitometer (Bio-Rad Laboratories Inc.). Bands were quantified by densitometry using the Quantity $\mathrm{One}^{\circledR}$ 1-D version 4.6.8 software (Bio-Rad Laboratories Inc.). Bovine serum albumin (\#05482, Sigma-Aldrich Co.) was used as a protein standard to draw a calibration curve, following the same procedure as OMVs samples. The protein concentration of the OMVs samples was determined by the interpolation from the standard curve. This protocol was optimized by Steeve Lima and Paulo Oliveira at i3S (unpublished).

\section{Proteomics Analysis}

A pellet of $5 \times 10^{11} \mathrm{H}$. pylori 26695 OMVs isolated from liquid bacterial cultures at 48, 64, and $72 \mathrm{~h}$ of growth was lysed in cold lysis buffer (1\% NP-40, $1 \%$ Triton X100 diluted in PBS, pH7.4) containing $1 \times$ Bacterial Protease Arrest $^{\mathrm{TM}}$ (GBiosciences, St. Louis, MO, United States) and $6 \mathrm{mg} / \mathrm{mL}$ lysozyme (PanReac AppliChem S. L. U., Barcelona, Spain), for $1 \mathrm{~h}$ on ice. Upon centrifugation $(21,000 \times g$ for $15 \mathrm{~min}$ at $4^{\circ} \mathrm{C}$ ), the cleared lysate was recovered and solubilized in a solution of $100 \mathrm{mM}$ Tris $\mathrm{pH} 8.5,1 \%$ sodium deoxycholate, $10 \mathrm{mM}$ tris(2-carboxyethyl)phosphine (TCEP), $40 \mathrm{mM}$ chloroacetamide and $1 \times$ complete $^{\mathrm{TM}}$ protease inhibitor cocktail (Roche Applied Science, Mannheim, Germany), for $10 \mathrm{~min}$, at $95^{\circ} \mathrm{C}$, with a constant rotation of $1,000 \mathrm{rpm}$ (Thermomixer, Eppendorf, Hamburg, Germany). Samples were processed for proteomics following the solid-phase-enhanced sample-preparation (SP3) protocol as described in Hughes et al. (2019). Briefly, enzymatic digestion was achieved by adding $2 \mu \mathrm{g}$ Trypsin/LysC to each sample and incubated overnight at $37^{\circ} \mathrm{C}$ with constant rotation (1,000 rpm). Protein identification and quantitation was performed by nanoLC-MS/MS using an Ultimate 3000 liquid chromatography system coupled to a Q-Exactive Hybrid Quadrupole-Orbitrap mass spectrometer (Thermo Fisher Scientific). Samples were loaded onto a trapping cartridge (Acclaim PepMap C18 100Å, $5 \mathrm{~mm} \times 300 \mu \mathrm{m}$ i.d., 160454, Thermo Fisher Scientific) in a mobile phase of $2 \%$ acetonitrile (ACN), $0.1 \%$ formic acid (FA) at $10 \mu \mathrm{L} / \mathrm{min}$. After 3 min loading, the trap column was switched in-line to a $50 \mathrm{~cm}$ by $75 \mu \mathrm{m}$ inner diameter EASY-Spray column (ES803, PepMap RSLC, C18, $2 \mu \mathrm{m}$, Thermo Fisher Scientific), at 300 $\mathrm{nL} /$ min. Separation was generated by gradient mixing A $(0.1 \%$ $\mathrm{FA})$ and $\mathrm{B}(80 \% \mathrm{ACN}, 0.1 \% \mathrm{FA})$ as follows: $5 \mathrm{~min}(2.5 \% \mathrm{~B}$ to $10 \% \mathrm{~B}), 120 \mathrm{~min}(10 \% \mathrm{~B}$ to $30 \% \mathrm{~B}), 20 \mathrm{~min}(30 \% \mathrm{~B}$ to $50 \% \mathrm{~B}$ ), $5 \mathrm{~min}(50 \% \mathrm{~B}$ to $99 \% \mathrm{~B}$ ), and $10 \mathrm{~min}$ (hold $99 \%$ B). Subsequently, the column was equilibrated with $2.5 \%$ B for $17 \mathrm{~min}$. Data acquisition was controlled by Xcalibur 4.0 and Tune 2.9 software (Thermo Fisher Scientific). The mass spectrometer was operated in data-dependent (dd) positive acquisition mode alternating between a full scan (m/z 380-1,580) and subsequent
HCD MS/MS of the 10 most intense peaks from full scan (normalized collision energy of 27\%). ESI spray voltage was $1.9 \mathrm{kV}$. Global settings: use lock masses best (m/z 445.12003), lock mass injection Full MS, chrom. peak width (FWHM) 15s. Full scan settings: 70k resolution (m/z 200), AGC target $3 \times 10^{6}$, maximum injection time $120 \mathrm{~ms}$. dd settings: minimum AGC target $8 \times 10^{3}$, intensity threshold $7.3 \times 10^{4}$, charge exclusion: unassigned, $1,8,>8$, peptide match preferred, exclude isotopes on, dynamic exclusion $45 \mathrm{~s}$. MS2 settings: microscans 1, resolution 35k (m/z 200), AGC target $2 \times 10^{5}$, maximum injection time $110 \mathrm{~ms}$, isolation window $2.0 \mathrm{~m} / \mathrm{z}$, isolation offset $0.0 \mathrm{~m} / \mathrm{z}$, spectrum data type profile. Three biological replicates were used for each time point and the LC-MS acquisition of each sample was performed in triplicate.

\section{Database Searching, Protein Identification, and Classification}

Raw data was processed using the Proteome Discoverer 2.5.0.400 software (Thermo Fisher Scientific) and searched against the UniProt ${ }^{1}$ database for the $H$. pylori 26695 Proteome 2021_01 release, 1552 entries, and a common contaminant database from MaxQuant (version 1.6.2.6, Max Planck Institute of Biochemistry, Munich, Germany). The Sequest HT and the MS Amanda 2.0 search engines, together with the Inferys PSM rescoring node, were used to identify tryptic peptides. The ion mass tolerance was $10 \mathrm{ppm}$ for precursor ions and $0.02 \mathrm{Da}$ for fragment ions. Maximum allowed missing cleavage sites was set to 2. Cysteine carbamidomethylation was defined as constant modification. Methionine oxidation, asparagine and glutamine deamidation, peptide $\mathrm{N}$-terminus glutamine to pyro-glutamine, and protein $\mathrm{N}$-terminus acetylation, methionine loss, and methionine loss plus acetylation, were defined as variable modifications. Peptide confidence was set to high. The processing node Percolator was enabled with the following settings: maximum delta $\mathrm{Cn}$ 0.05 ; decoy database search target false discovery rate $1 \%$, validation based on q-value. Protein label free quantitation was performed with the Minora feature detector node at the processing step. Precursor ions quantification was performed at the processing step with the following parameters: unique plus razor peptides were used for quantification, precursor abundance based on intensity and normalization based on total peptide amount. Common contaminants were excluded from data analysis. The ANOVA hypothesis test (individual proteins) for $p$-value calculation was performed for the three bacterial growth periods. Differentially expressed proteins were identified using the following parameters: fold change ratios \pm 2.00 and $p<0.05$. The total abundance of the predicted groups was calculated by the sum of the abundance of proteins classified for each group Prediction of proteins cellular localization and molecular function was obtained from the gene ontology (GO) UniProt database (see text foot note 1). The mass spectrometry proteomics data have been deposited to the ProteomeXchange Consortium via the PRIDE (Perez-Riverol et al., 2019) partner repository with the dataset identifier PXD025393 and 10.6019/PXD025393.

${ }^{1}$ www.uniprot.org 


\section{Statistical Analysis}

Data were analyzed using the GraphPad Prism version 8.4.3 software (San Diego, CA, United States). The one-, twoway and Brown-Forsythe ANOVA, with the post hoc Tukey's, Dunnett's or Sidak's tests for paired comparisons, were applied for comparisons between three independent groups. Statistically significance was set at $p$-values $\leq 0.05\left({ }^{*} p \leq 0.05,{ }^{* *} p \leq 0.01\right.$, *** $\left.p \leq 0.001,{ }^{* * * *} p \leq 0.0001\right)$.

\section{RESULTS}

\section{Helicobacter pylori Growth and Viability in F12 Liquid Medium Supplemented With Cholesterol}

Considering the goal to isolate OMVs from $H$. pylori cultures grown in a chemically defined medium, we started by characterizing the bacterial growth and viability in the Ham's F-12 liquid medium supplemented with cholesterol, previously reported to support $H$. pylori growth (Testerman et al., 2001).

The bacterial growth of $H$. pylori 26695 was monitored by measuring the optical density at $600 \mathrm{~nm}\left(\mathrm{OD}_{600}\right)$ of the bacterial liquid cultures at $24,48,64$, and $72 \mathrm{~h}$, starting with an inoculum density of 0.02 per $\mathrm{mL}$ at $\mathrm{OD}_{600}\left(\sim 6 \times 10^{6} \mathrm{CFUs} / \mathrm{mL}\right)$ in $200 \mathrm{~mL}$ of F12-cholesterol medium (Figure 1A). The growth curve was predicted using the Gompertz model (Mytilinaios et al., 2012) fitted to our data. H. pylori presented an exponential growth during the first $32 \mathrm{~h}$ of culture, after which it reached a stationary phase that lasted nearly until the endpoint of $72 \mathrm{~h}$. The experimental $\mathrm{OD}_{600}$ values at $24 \mathrm{~h}, 48 \mathrm{~h}, 64 \mathrm{~h}$, and $72 \mathrm{~h}$ were, respectively, $0.127 \pm 0.004,0.147 \pm 0.003,0.148 \pm 0.004$, and $0.139 \pm 0.003$, decreasing $6.1 \%$ from $64 \mathrm{~h}$ to $72 \mathrm{~h}$, although not statistically significant ( $p=0.3528$; one-way ANOVA with post hoc Tukey's test), indicating that bacterial growth might become limited by nutrient availability. The growth kinetics of H. pylori 26695 in the chemically defined F12-cholesterol medium and in the complex BB medium supplemented with $5 \%$ FBS was compared, under the same experimental conditions (Supplementary Figure 1A). The growth curve was similar for both media, although bacteria grew faster in BB-FBS, with bacterial cultures reaching higher densities $\left(\mathrm{OD}_{600}\right.$ at $24 \mathrm{~h}$ : $0.160 \pm 0.010 ; 48$ h: $0.199 \pm 0.004 ; 64$ h: $0.206 \pm 0.011$; and $72 \mathrm{~h}:$ $0.209 \pm 0.005)$.

To assess the viability of $H$. pylori 26695 strain in F12cholesterol, we determined the number of CFUs and performed the LIVE/DEAD BacLight Bacterial Viability assay using flow cytometry. The number of CFUs increased until $48 \mathrm{~h}$ of bacterial growth $\left(24 \mathrm{~h}: 9.46 \pm 0.96 \times 10^{7} \mathrm{CFUs} / \mathrm{mL} ; 48 \mathrm{~h}\right.$ : $\left.1.12 \pm 0.06 \times 10^{8} \mathrm{CFUs} / \mathrm{mL}\right)$, and decreased slightly at $64 \mathrm{~h}$, although not statistically significant $\left(64 \mathrm{~h}: 9.11 \pm 0.71 \times 10^{7}\right.$ CFUs/mL; $48 \mathrm{~h}$ vs. $64 \mathrm{~h}: p=0.2080)$, and until $72 \mathrm{~h}$ $\left(6.38 \pm 0.66 \times 10^{7} \mathrm{CFUs} / \mathrm{mL}, 48 \mathrm{~h}\right.$ vs. $72 \mathrm{~h}: p=0.0082 ; 64 \mathrm{~h}$ vs. 72 h: $p=0.0571$ ) (Figure 1B). For comparative purposes, we also evaluated the number of CFUs in BB-FBS bacterial cultures (Supplementary Figure 1B). The number of CFUs was similar at $24 \mathrm{~h}$ of growth in both media $\left(24 \mathrm{~h}: 8.47 \pm 0.38 \times 10^{7}\right.$
CFUs/mL; $p=0.8217)$, and significantly lower in BB-FBS at $48 \mathrm{~h}$ $\left(6.88 \pm 0.44 \times 10^{7} \mathrm{CFUs} / \mathrm{mL} ; p<0.0001\right), 64 \mathrm{~h}\left(4.58 \pm 0.71 \times 10^{7}\right.$ CFUs/mL; $p<0.0001)$, and $72 \mathrm{~h}\left(3.00 \pm 0.70 \times 10^{7} \mathrm{CFUs} / \mathrm{mL}\right.$; $p=0.0030)$. These results show that F12-cholesterol medium sustains the growth and a higher number of viable $H$. pylori, although at a slower rate than BB-FBS medium. The viability of H. pylori 26695 grown in F12-cholesterol was also evaluated by another method, the LIVE/DEAD BacLight assay. This assay relies on the simultaneous staining with two fluorescent nucleic acids dyes to distinguish between live and dead bacteria: SYTO9 (green) that enters in all bacterial cells, staining both live and dead cells, and PI (red) that enters in cells with a disrupted membrane, labeling only dead bacteria. Using SYTO9 to define the bacterial cell population, the percentage of live $\left(\mathrm{SYTO}^{+} \mathrm{PI}^{-}\right)$and dead $\left(\mathrm{SYTO}^{+} \mathrm{PI}^{+}\right.$) bacteria was then calculated for each sample. No statistically significant differences in the percentage of live ( $48 \mathrm{~h}$ : $93.3 \pm 1.8 \% ; 64$ h: $92.8 \pm 1.6 \% ; 72$ h: $91.0 \pm 1.7 \%)$ and dead (48 h: $6.7 \pm 1.8 \%$; 64 h: $7.1 \pm 1.6 \%$; 72 h: $8.7 \pm 1.7 \%$ ) bacteria were found between the experimental time points (Figure 1B and Supplementary Figure $\mathbf{2}$ for gating strategy). This shows that bacteria remained viable until $72 \mathrm{~h}$, despite the above-mentioned decrease in the number of CFUs.

Knowing that $H$. pylori morphology changes from a bacillary to a coccoid structure in response to nutrient deprivation and other adverse environmental conditions (Kusters et al., 1997; Azevedo et al., 2007), which could explain the differences between CFUs counts and LIVE/DEAD assay results, we evaluated the morphological alterations during the bacterial growth in F12cholesterol. Bacillary, U-shaped, and coccoid bacteria were manually counted from negative stain TEM micrographs taken from 48, 64, and $72 \mathrm{~h}$ bacterial liquid cultures (Figure 1C). No statistically significant differences were found regarding the frequency of each morphological shape between samples collected at 48 and $64 \mathrm{~h}$, and between 64 and $72 \mathrm{~h}$ samples (Figure 1D). However, a significant decrease in the number of bacillary $(p=0.003)$ and an increase in the number of coccoid H. pylori $(p=0.012)$ were observed in samples collected at $72 \mathrm{~h}$ when compared to those obtained at $48 \mathrm{~h}$. This observation suggests that coccoid forms are viable but non-culturable (Ierardi et al., 2020), as there was no alteration in the frequency of viable bacteria, assessed by the LIVE/DEAD assay, at this time point. As an ancillary analysis, we checked the morphology of $H$. pylori grown in F12-cholesterol by SEM (Supplementary Figure 3), which confirmed that the bacillary shape was predominant at all time points, with the U-shape and coccoid bacteria becoming noticeable in the $72 \mathrm{~h}$ cultures.

Altogether, these results show that the F12-cholesterol medium is capable of nutritionally support $H$. pylori growth, preserving its typical bacillary morphology and viability.

\section{Quantification and Morphological Characterization of OMVs Secreted by Helicobacter pylori Grown in F12-Cholesterol Medium}

After ensuring that the F12-cholesterol medium supports both growth and viability of $H$. pylori 26695 under our experimental 


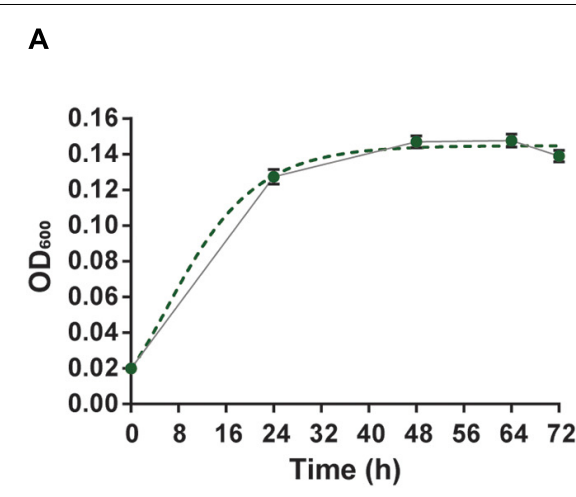

C

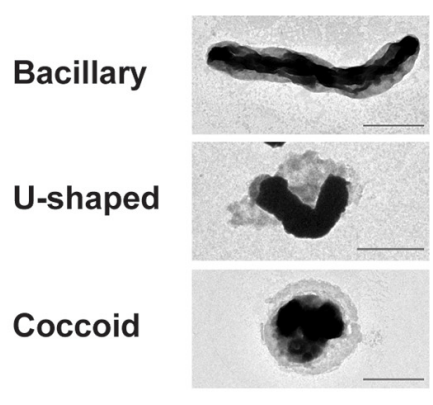

B

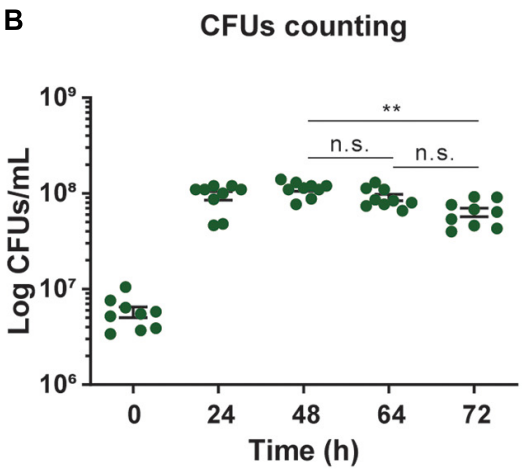

\section{LIVE/DEAD assay}

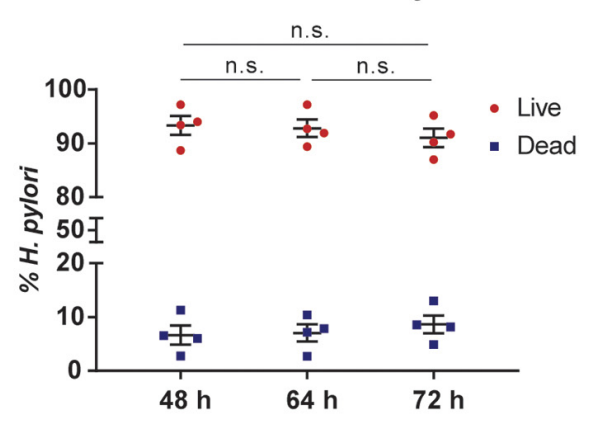

D

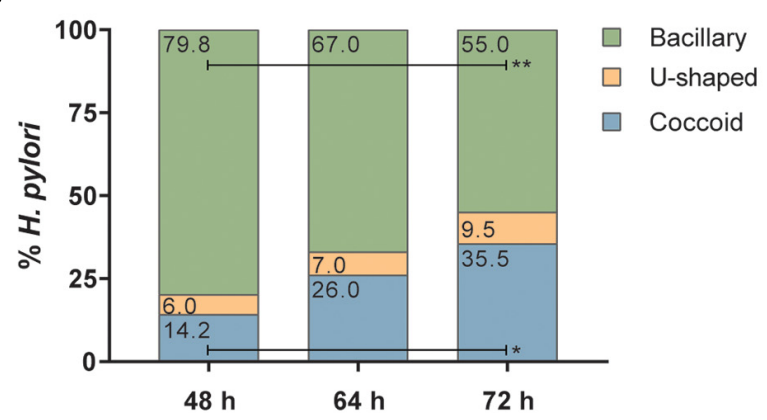

FIGURE 1 | Kinetics, viability, and morphology of Helicobacter pylori growth in liquid F-12-cholesterol. (A) Growth curve of Helicobacter pylori 26695 grown in F12 liquid medium supplemented with $1 \times$ cholesterol (continuous line), modeled using the Gompertz growth equation model (dotted line), calculated with GraphPad Prism, and based on the optical density measurements of the bacterial suspension at $600 \mathrm{~nm}\left(\mathrm{OD}_{600}\right)$ from $24,48,64$, and $72 \mathrm{~h}$ cultures. Data are shown as mean \pm SEM of 16 biological replicates. (B) Bacterial viability evaluated by colony-forming units (CFUs) counting (left graph) and the LIVE/DEAD BacLight Bacterial Viability assay (right graph). The number of CFUs was determined at the referred time points, represented as CFUs/mL. Each dot represents a biological replicate $(n=9)$ and data are shown as mean \pm SEM. Statistical significance was evaluated using the one-way ANOVA with post hoc Tukey's test, comparing all datasets with each other; only comparisons between 48, 64, and 72 h were illustrated for the sake of simplicity; ${ }^{* *} p \leq 0.01$, n.s. - not significant. LIVE/DEAD BacLight Bacterial Viability assay at 48, 64, and 72 h of growth, by flow cytometry. After sample staining with SYTO9 and PI dies and acquisition on a FACSCanto II cytometer, live and dead bacteria were gated and defined as SYTO9 ${ }^{+} \mathrm{PI}^{-}$and SYTO9 ${ }^{+} \mathrm{PI}^{+}$, respectively. Each dot represents a biological replicate $(n=4)$ and data are shown as mean \pm SEM of the frequency of gated SYTO9 ${ }^{+} \mathrm{PI}^{-}$and SYTO9 ${ }^{+} \mathrm{Pl}^{+}$bacteria. Statistical significance was evaluated using the two-way ANOVA with post hoc Tukey's test; n.s. - not significant. (C) Representative negative stain TEM micrographs of bacillary, U-shaped, and coccoid forms of $H$. pylori from a 64 h liquid culture. Scale bars: $1 \mu \mathrm{m} ; 80,000 \times$ (bacillary and coccoid) and 8,000× (U-shaped) original magnifications. (D) Quantification of bacillary, U-shaped, and coccoid forms of $\mathrm{H}$. pylori present in F12-cholesterol liquid cultures at 48,64, and $72 \mathrm{~h}$ from negative stained TEM micrographs, using manual counting. The frequency of each bacterial form was calculated considering the total number of bacteria $(741,1,215$, and 1,061) counted in micrographs taken from samples of each time point, $48 \mathrm{~h}(n=5), 64 \mathrm{~h}(n=5)$, and $72 \mathrm{~h}(n=2)$, respectively. The mean frequency of each form is displayed inside the respective bar. Statistical significance was evaluated using the two-way ANOVA with post hoc Tukey's test, comparing all datasets with each other; statistical significance was only observed between 72 and $48 \mathrm{~h}$ for bacillary and coccoid forms; ${ }^{*} p \leq 0.05$ and ${ }^{* *} p \leq 0.01$.

settings, we optimized the OMVs' isolation protocol to be fast and simple, while reliable. This protocol comprises one low-speed centrifugation followed by a $0.45 \mu \mathrm{m}$-filtration to deplete both bacterial cells and debris, one ultracentrifugation to recover OMVs, and a final ultracentrifugation to wash the OMVs fraction (Figure 2). The overall duration of the protocol is of approximately $4 \mathrm{~h}$, distributed in $60 \mathrm{~min}$ of hands-on and $195 \mathrm{~min}$ of hands-off time. This protocol is substantially shorter than those published, which report the hands-off time between $335 \mathrm{~min}$ to 1,170 min (Mullaney et al., 2009; Olofsson et al., 2010).

The OMVs recovered following this protocol exhibited a spherical shape with a central depression and a heterogeneous size, as visualized by negative staining followed by TEM (Figure 3A), matching the OMVs' prototypical morphology and size distribution (Chutkan et al., 2013). Moreover, ultrastructural analysis enabled validation of the vesicles as OMVs, since they are delimited by a single lipid bilayer (Figure 3B). TEM analysis revealed the absence of bacterial debris, flagella, and proteins, showing that OMVs preparations were highly pure. The comparative analysis of the negative staining images of OMVs isolated from $H$. pylori 26695 grown in F12-cholesterol and in complex BB-FBS medium, and between the respective culture media alone, highlighted the importance of using the synthetic medium for the recovery of highly pure OMVs. F12cholesterol medium and OMVs isolated from F12-cholesterol bacterial cultures had a clear background, without protein aggregates (Figure 3A and Supplementary Figure 4A), distinctly from BB-FBS medium and the respective OMVs samples, in which the presence of proteins was detected (Supplementary Figures 4B,C).

We next characterized the size distribution and number of OMVs recovered from the 48,64 , and $72 \mathrm{~h} \mathrm{H}$. pylori cultures by 


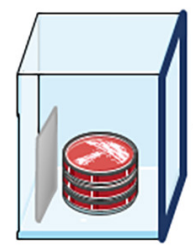

H. pylori solid culture in TSA plates

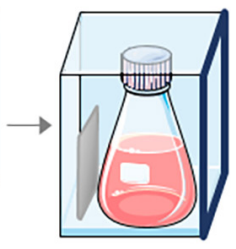

H. pylori liquid culture in F12-cholesterol $\left(\mathrm{OD}_{600}\right.$ 0.02)
$48 \mathrm{~h}$

$64 \mathrm{~h}$

$72 \mathrm{~h}$

Low-speed

centrifugation

$15,000 \times \mathrm{g}$

$15 \mathrm{~min}$ at $4^{\circ} \mathrm{C}$

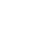
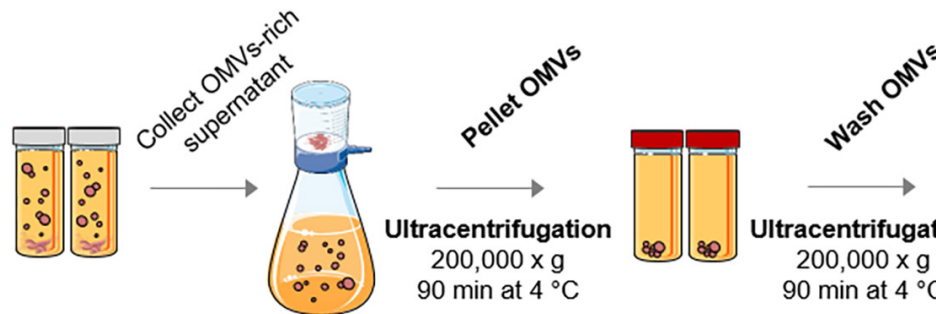

Uit

racentrifugation

$200,000 \times \mathrm{g}$

90 min at $4{ }^{\circ} \mathrm{C}$

Supernantant

$0.45 \mu \mathrm{m}$-filtration

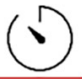

Hands-on: $60 \mathrm{~min}$ Hands-off: 195 min

FIGURE 2 | Outline of the OMVs isolation method from Helicobacter pylori cultures in F12-cholesterol medium. After the establishment of the liquid bacterial culture, the protocol for OMVs isolation includes one low-speed centrifugation, a $0.45 \mu \mathrm{m}$-filtration of the bacteria-free supernatant, and two ultracentrifugations for the recovery and wash of OMVs, comprising $60 \mathrm{~min}$ of hands-on period and $195 \mathrm{~min}$ of hands-off period (see details in Materials and Methods section). This figure used elements from Servier Medical Art (www.servier.fr/servier-medical-art).

NTA (Figure 3C). More than 99\% of all OMVs presented a size range between 20 and $200 \mathrm{~nm}$, regardless of the culture time, and $0.66 \%$ of vesicles were detected above the $200 \mathrm{~nm}$. The presence of OMVs higher than $450 \mathrm{~nm}$ was negligible, which matched the exclusion filter pore-size and demonstrated the absence of vesicle aggregates. From the NTA data, we also identified that the mode sizes of OMVs isolated from the 48, 64, and $72 \mathrm{~h}$ liquid cultures were, respectively, $84.3 \pm 1.7,79.8 \pm 6.4$, and $83.3 \pm 5.0 \mathrm{~nm}$. Despite the fact that OMVs isolated from $48 \mathrm{~h}$ bacterial cultures were more heterogeneous in size than OMVs from 64 and $72 \mathrm{~h}$ cultures, presenting a distinctive population of smaller OMVs with a diameter ranging from 15 to $60 \mathrm{~nm}$, the size distribution of OMVs from the three time points presented no statistically significant differences between them.

Concerning the yield of OMVs, an average of $1.68 \pm 0.24 \times 10^{10}, 3.37 \pm 0.31 \times 10^{10}$, and $3.59 \pm 0.91 \times 10^{10}$ OMVs per $\mathrm{mL}$ of liquid culture was recovered from the 48 , 64, and $72 \mathrm{~h}$ bacterial cultures, respectively (Figure 3D). The $64 \mathrm{~h}$ bacterial cultures produced a significantly higher number of OMVs/mL when compared to the $48 \mathrm{~h}$ cultures $(p=0.014)$, but not significantly different from the $72 \mathrm{~h}$ cultures. Although we cannot exclude some loss of OMVs during the isolation procedure, we assume that the number of secreted vesicles is likely near the recovered ones, given that no vesicles were detected in the supernatant collected after the first ultracentrifugation, both in F12-cholesterol and in BB-FBS, when analyzed by TEM (Supplementary Figures 4D,E). As so, the shorter number of steps and the recovery of a high yield of vesicles emphasize the efficacy of our protocol, even when different culture media are used.

To ascertain the applicability of this protocol to $H$. pylori strains other than 26695, we selected two additional $H$. pylori reference strains, 60190 and Tx30a. These strains were grown in F12-cholesterol medium, under the same conditions as strain 26695, and their OMVs were isolated and characterized by TEM and NTA, in the $64 \mathrm{~h}$ bacterial cultures. OMVs recovered from H. pylori 60190 and TX30a were highly pure and with the same size heterogeneity as 26695-OMVs (Figures 3E,F). The number of OMVs recovered per $\mathrm{mL}$ of bacterial culture was similar between the three strains at $64 \mathrm{~h}$ of bacterial growth (26695: $3.37 \pm 6.27 \times 10^{9} ; 60190: 3.09 \pm 1.29 \times 10^{10}$; Tx30a: $2.45 \pm 1.01 \times 10^{10} \mathrm{OMVs} / \mathrm{mL}$ ) (Figure 3G).

In summary, these results show that OMVs recovered from H. pylori grown in F12-cholesterol cultures are bona fide, presenting the same morphology and ultrastructure as those isolated from complex media (Chutkan et al., 2013; Turner et al., 2018). Furthermore, our abridged protocol provided a good yield and a highly pure population of OMVs from various $H$. pylori strains and in different phases of bacterial growth.

\section{Proteomic Content and Proteomic Analysis of OMVs Isolated From Helicobacter pylori 26695 Grown in F12-Cholesterol Culture Medium}

The next aim was to characterize and compare the protein cargo of OMVs secreted by $H$. pylori grown in F12-cholesterol at different time points of bacterial growth. The total protein amount from OMVs was assessed by SDS-PAGE and gel staining with BlueSafe, using lysates from $10^{11}$ OMVs (Figure 4). OMVs isolated from 48, 64 and $72 \mathrm{~h}$ bacterial cultures presented an identical protein profile (Figure 4A) and similar total protein amounts (48 h: $3.55 \pm 1.16 ; 64 \mathrm{~h}: 4.02 \pm 0.95 ; 72 \mathrm{~h}: 4.25 \pm 1.42 \mu \mathrm{g}$ of protein per $10^{11} \mathrm{OMVs}$ ) (Figure 4B).

Next, we applied nanoscale liquid chromatography coupled to tandem mass spectrometry (nanoLC-MS/MS) to $5 \times 10^{11}$ OMVs of each time point of bacterial growth, and analyzed the differential expression of proteins between each of them. A total of 267, 268 and 269 proteins from $H$. pylori 26695 were identified on 48, 64, and 72 h-OMVs, respectively (Supplementary Table 1). Of these, 233, 231, and 232 proteins with a false discovery rate of $1 \%$ were further classified according to their predicted cellular localization, biological process and molecular function, using the GO UniProt database resource and manual curation (Supplementary Table 2). All proteins identified in 64 h-OMVs (231) were common to 48 and $72 \mathrm{~h}$ samples. 

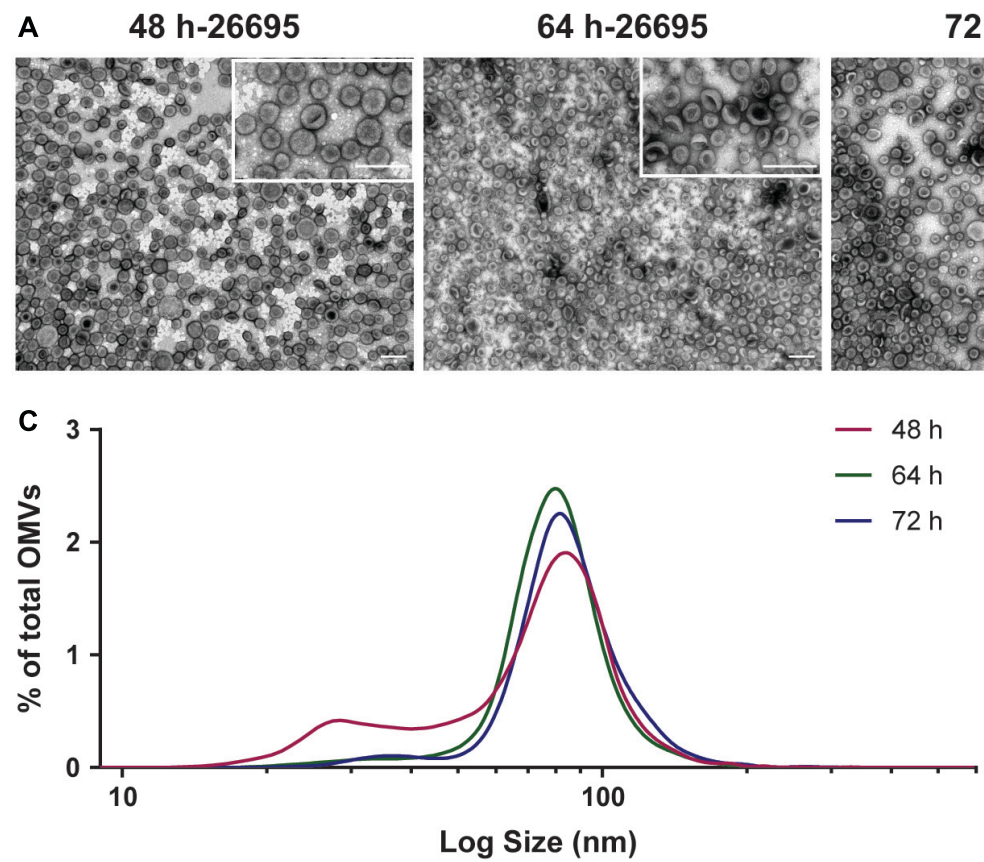

E
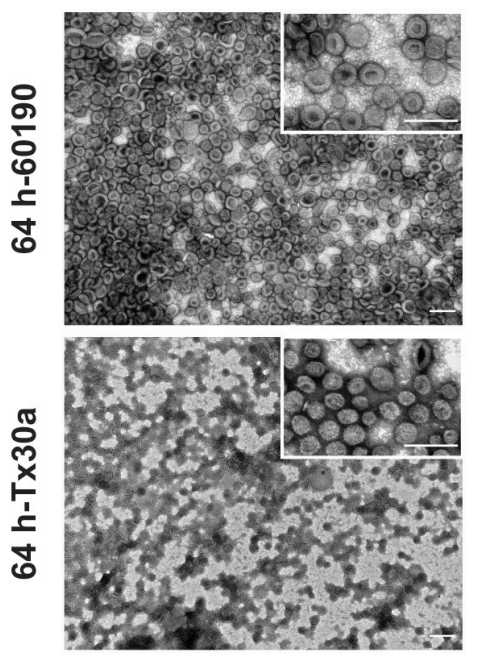

64 h-26695

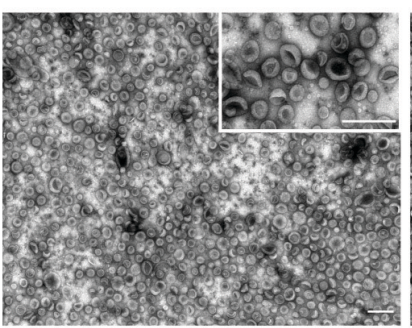

F

G
72 h-26695

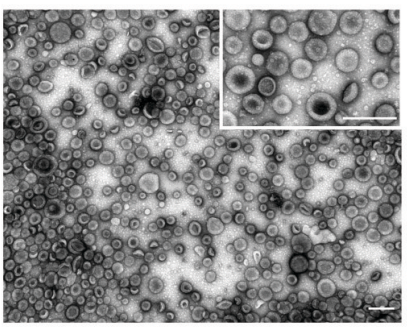

B

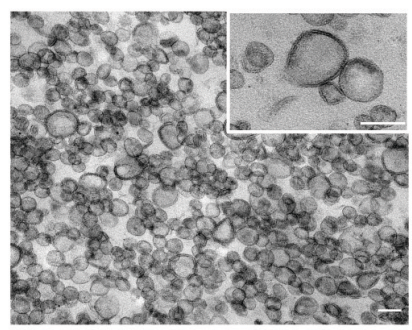

n. S.

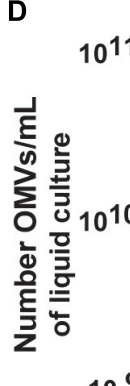

D

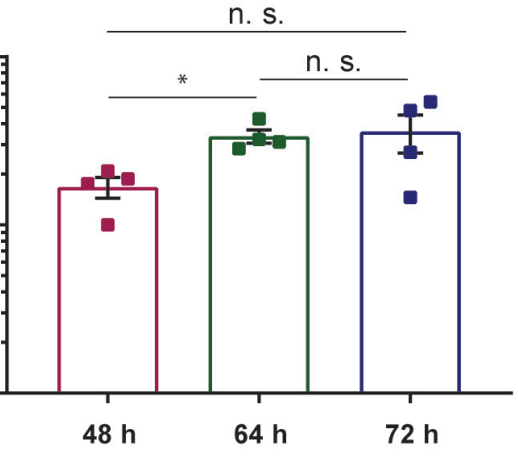

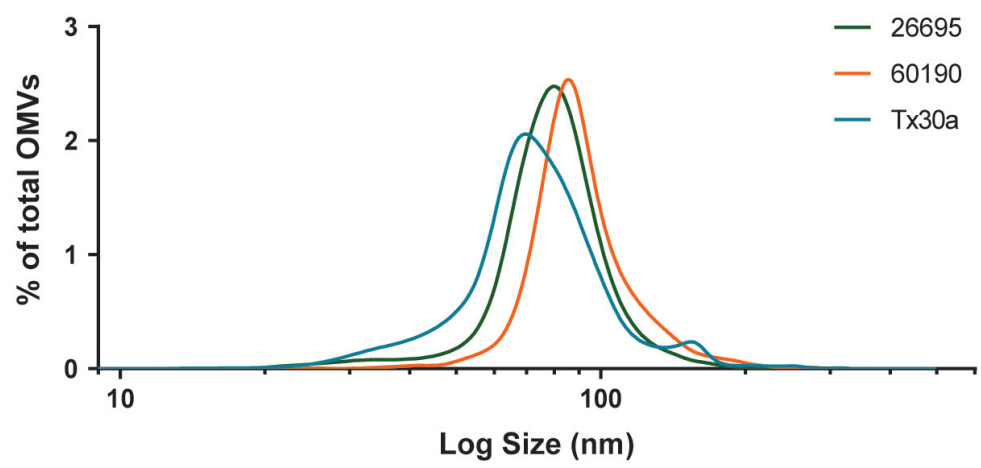

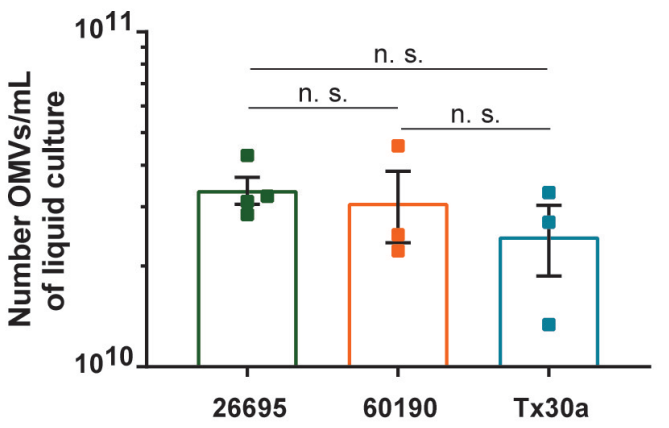

FIGURE 3 | Morphological characterization, size distribution, and yield of OMVs secreted by Helicobacter pylori grown in F12-cholesterol medium. (A) Negative staining of OMVs isolated from $\mathrm{H}$. pylori $26695 \mathrm{~F} 12$-cholesterol liquid cultures at 48, 64, and $72 \mathrm{~h}$ of growth, and (B) ultrastructure section of OMVs from $64 \mathrm{~h}$ H. pylori cultures. Scale bars: $200 \mathrm{~nm}$; 50,000×, 100,000× [insets in panel (A)] and 200,000× [inset in panel (B)] original magnifications. (C) Size distribution, represented as percentage of the total number of isolated OMVs; data are shown as mean \pm SEM of four biological replicates and statistical significance was evaluated using the one-way ANOVA with post hoc Tukey's test; n.s. - not significant. (D) Number of recovered OMVs per mL of bacterial culture, determined using Nanoparticle Tracking Analysis (NTA) at 48, 64, and $72 \mathrm{~h}$ periods of bacterial growth; data are shown as mean \pm SEM of four biological replicates and statistical significance was evaluated using the one-way ANOVA with post hoc Tukey's test; ${ }^{\star} p \leq 0.05$, n.s. - not significant. (E) Representative negative stain TEM micrographs of H. pylori 60190 and Tx30a-OMVs generated from 64 h F12-cholesterol bacterial cultures. Scale bars: 200 nm; 50,000× and 100,000× (insets) original magnification. (F) Size distribution, represented as percentage of the total number of isolated OMVs at $64 \mathrm{~h}$ of liquid culture, and (G) number of recovered OMVs per $\mathrm{mL}$ of bacterial culture determined by NTA; data are shown as mean \pm SEM of 4 (for 26695) or 3 (for 60190 and Tx30a) biological replicates and statistical significance was evaluated using the Brown-Forsythe and Welch ANOVA with post hoc Dunnett's test; n.s. - not significant. 
A

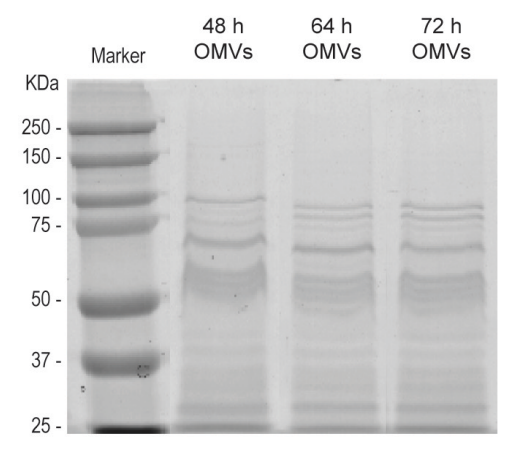

D

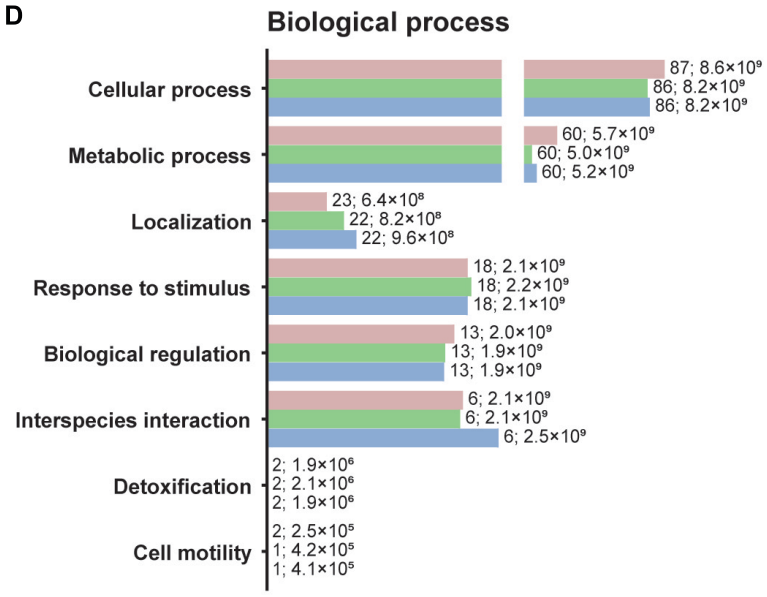

B

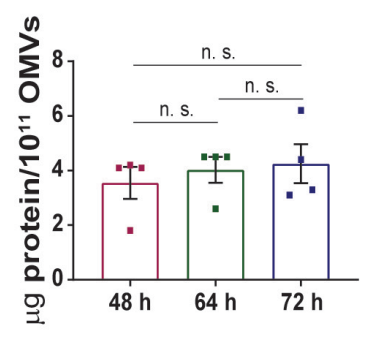

C

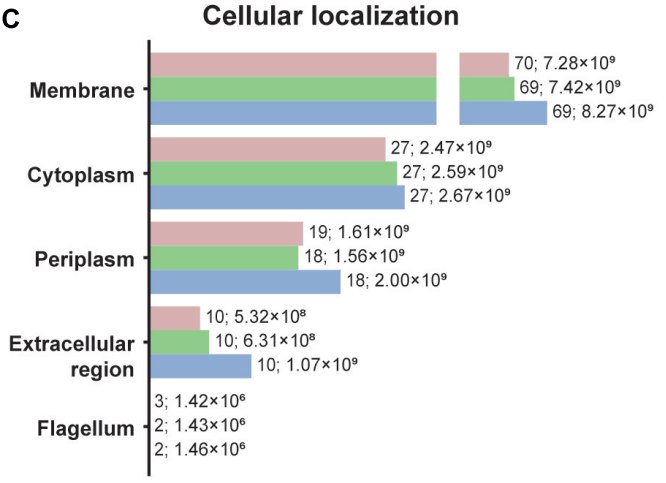

E

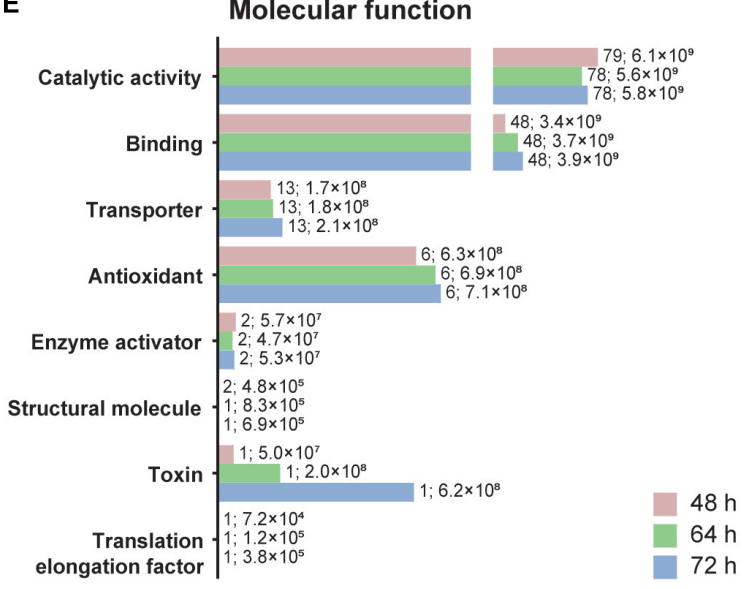

FIGURE 4 | Protein profile and proteomic analysis of OMVs secreted by Helicobacter pylori grown in F12-cholesterol medium. (A) Protein profile of $10^{11}$ OMVs isolated from 48, 64, and $72 \mathrm{~h}-\mathrm{H}$. pylori 26695 bacterial cultures, after staining a SDS-PAGE with BlueSafe, and corresponding (B) protein quantification. Data are shown as mean \pm SEM of four biological replicates and statistical significance was evaluated using the one-way ANOVA with post hoc Tukey's test; $n$.s. - not significant. Proteomic analysis of 48, 64, and $72 \mathrm{~h}$-OMVs isolated from H. pylori 26695 F12-cholesterol liquid cultures by nanoLC-MS/MS and prediction of the (C) cellular localization, (D) biological process, and (E) molecular function of the identified proteins using the gene ontology UniProt database. Data are shown as the abundance of proteins in each group; for each bar, the number of proteins and respective abundance is indicated.

Pyruvate ferredoxin oxidoreductase was identified only in 48 and $72 \mathrm{~h}$ samples, whereas flagellar P-ring protein was detected exclusively in $48 \mathrm{~h}$-OMVs.

In terms of cellular localization (Figure 4C), the most diverse and abundant group of proteins identified in OMVs from all time points analyzed was predicted to be associated with the bacterial membrane, and within this group outer membrane proteins (OMPs) were the most frequent (48 h: $n=50$, abundance $=5.67 \pm 0.41 \times 10^{9}$; $64 \mathrm{~h}: n=49$, abundance $=5.73 \pm 1.25 \times 10^{9} ; 72 \mathrm{~h}: n=49$, abundance $\left.=6.52 \pm 0.74 \times 10^{9}\right)$. Periplasmic, cytoplasmic and extracellular region-associated proteins were also detected. In addition, 3 flagellar proteins were identified in $48 \mathrm{~h}$ samples, while only 2 were detected in 64 and 72 h-OMVs. Considering that OMVs originate from the blebbing of the OM, a high abundance of membrane-associated proteins was expected, and is in agreement with previous proteomic data from H. pylori 26695 OMVs (Zavan et al., 2019).

Concerning the biological process analysis, proteins were distributed into eight groups (Figure 4D). Proteins involved in cellular processes constituted the most numerous and abundant group, followed by proteins involved in metabolic processes, localization proteins, response to stimulus, biological regulation, and interspecies interaction proteins. Detoxification and cell motility proteins were the less abundant.

Regarding the molecular functions (Figure 4E), the majority of the classified proteins was predicted to have catalytic, binding and/or transporter activities, followed by proteins with antioxidant and structural molecular activities, enzyme activators, a toxin, and a translation elongation factor. Besides the above-mentioned molecular functions, several of the identified proteins are also involved in processes related with $H$. pylori colonization, survival, and pathogenesis. In particular, urease $\alpha / \beta$ neutralizes the acidic environment of the gastric mucosa (Marshall et al., 1990), which together with outer membrane proteins (BabA and OipA), contributes to H. pylori colonization (Ishijima et al., 2011; Teymournejad et al., 2017); $\beta$-lactamase (HcpA, HcpC, HcpD, and HcpE) provides resistance to amoxicillin (Tseng et al., 2009); HtrA disrupts the epithelial cell-cell junctions, enabling transmigration of $H$. pylori across 
the gastric epithelium (Tegtmeyer et al., 2017); VacA and GGT induce, vacuolization and apoptosis (Ricci et al., 1997; Shibayama et al., 2003); HP-NAP, OipA, and urease $\beta$ act as immune modulators by inducing pro-inflammatory responses (Yamaoka et al., 2002; de Bernard and D'Elios, 2010; Schoep et al., 2010); catalase, superoxide dismutase and thioredoxin protect $H$. pylori from the oxidative stress (Pesci and Pickett, 1994; Odenbreit et al., 1996; Kuhns et al., 2015); HP-NAP and bacterial nonheme ferritin are iron storage proteins, a key element for bacterial survival (Ge and Sun, 2012). The presence of these virulence factors in $\mathrm{H}$. pylori OMVs isolated from F12-cholesterol and from other complex liquid cultures (Mullaney et al., 2009; Olofsson et al., 2010; Zavan et al., 2019) denotes that some proteins may be preferably loaded onto OMVs, independently of the culture media or bacterial strain, and highlights the importance of OMVs for the success of $H$. pylori infection. Seventy of the 233 identified proteins were uncharacterized and approximately half of them did not have a predicted cellular localization or molecular function.

Although OMVs isolated from 48, 64, and 72 h-bacterial cultures had a similar protein diversity, some of the proteins were differentially expressed (Supplementary Table 3 for the comparison between 48 and $64 \mathrm{~h}$; Supplementary Table 4 for the comparison between 48 and 72 h; Supplementary Table 5 for the comparison between 64 and $72 \mathrm{~h}$ ). In particular, the virulence factor VacA was found to be significantly more abundant in 64 and $72 \mathrm{~h}$-OMVs, differently from the data of Zavan et al. (2019) that shows an enrichment of this protein in $16 \mathrm{~h}$-OMVs in comparison to vesicles isolated from $48 \mathrm{~h}$ and $72 \mathrm{~h}$ bacterial cultures. Additionally, the thioredoxin reductase, which protects H. pylori from the oxidative stress (Kuhns et al., 2015), and 2 proteins involved in iron acquisition and transport across the membrane, which is an essential micronutrient for bacterial survival, FrpB (Gonzalez-Lopez and Olivares-Trejo, 2009) and FecA, presented a significantly higher expression in 64 and $72 \mathrm{~h}-$ OMVs, in comparison to $48 \mathrm{~h}$ samples.

Overall, our proteomic analysis confirms the reliability of the protocol, by showing that the cargo of OMVs contain proteins from the membrane, periplasmic, and cytoplasmic bacterial compartments, and supports the selective sorting of protein cargo into OMVs, as only a fraction of the total bacterial proteins is represented in the cargo of OMVs.

\section{DISCUSSION}

Outer membrane vesicles have emerged as an important delivery system of bacterial components with an impact on bacteriabacteria and bacteria-host interactions. In addition, OMVs are increasingly used for diverse therapeutic applications, namely vaccine development, taking advantage of their flexibility to genetic manipulation and nanosized structure for dissemination throughout the body, besides their easy production at large-scale and reduced costs (Bitto and Kaparakis-Liaskos, 2017).

The prevailing methods for the isolation of $H$. pylori OMVs are time-consuming and diverse, hampering the standardization of a suitable protocol for OMVs isolation, which is essential for downstream research and biomedical applications, namely the assessment of the functional roles of OMVs in vitro and in vivo, and large-scale production. The current published protocols have various disadvantages. First, they rely on the use of complex undefined media that contain yeast and animal tissue extracts, thus affecting the purity, content, and variability of OMVs between samples (Olofsson et al., 2010; Tsolakos et al., 2010). Second, they have a lengthy duration due to the multiple centrifugation steps, which might compromise the final yield of OMVs (Chutkan et al., 2013). Finally, the high variability of procedures and diversity of protocols weakens the comparison between studies, as the recovered fractions of OMVs can be different.

To overcome these drawbacks, we developed an isolation method of $H$. pylori OMVs trimming non-essential steps after selecting F12 supplemented with cholesterol, a chemically defined medium, as the bacterial culture medium for $H$. pylori growth. The final layout of this method included one lowspeed centrifugation followed by supernatant $0.45 \mu \mathrm{m}$-filtration to remove bacteria and debris from the culture medium and to collect all range of OMVs sizes, and finally two ultracentrifugations for the recovery and washing of the OMVs.

Our results showed that the F12-cholesterol medium was effective in sustaining bacterial growth, while achieving higher viability than the complex BB-FBS medium. Like previously described for other bacterial culture media (Kusters et al., 1997; Adams et al., 2003; Olofsson et al., 2010), we observed a morphological change of $H$. pylori from the bacillary to the coccoid forms throughout bacterial growth. The significant decrease in the number of CFUs at $72 \mathrm{~h}$ was not mirrored by the loss of bacterial viability using the LIVE/DEAD assay, likely due to the fact that coccoid forms are viable but non-culturable bacteria, with minimal metabolic activity, and preserved membrane and genetic material integrities (Adams et al., 2003; Ierardi et al., 2020).

Associated with the bacterial growth was the increased number of secreted OMVs, in accordance with descriptions of OMVs isolated from complex liquid cultures (Olofsson et al., 2010; Zavan et al., 2019). Over 99\% of OMVs measured between 20 and $200 \mathrm{~nm}$, with mode of nearly $80 \mathrm{~nm}$, which is distinct from the enrichment in 100 to $200 \mathrm{~nm}$ sized OMVs isolated from $H$. pylori grown in BHI supplemented with $0.2 \% \beta$-cyclodextrin (Zavan et al., 2019). This difference might be related with the distinct medium used, as well as with the effect of $\beta$-cyclodextrin, which chelates cholesterol that is essential for $H$. pylori growth (Hutton et al., 2010; Jimenez-Soto et al., 2012), highlighting how bacterial growth conditions impact on the properties of OMVs. Our findings also demonstrated the reproducibility of this protocol, as OMVs isolated from different $H$. pylori strains presented a similar size heterogeneity and were equally pure.

OMVs isolated from F12-cholesterol medium using our shorter protocol were highly pure, free from protein aggregates, flagella, and other bacterial contaminants, while requiring significantly less hands-on/hands-off time. Of relevance was also the high yield of OMVs, with around $10^{10}$ OMVs per $\mathrm{mL}$ of bacterial culture. Moreover, the proteomic analysis of our OMVs confirmed that their cargo 
contained proteins from several bacterial compartments (e.g., membrane, periplasm, and cytoplasm) and with diverse biological functions, in particular known bacterial virulence factors, already described for different $H$. pylori strains (Jarzab et al., 2020), reinforcing their reliability. Besides the common proteins identified between our and other OMVs' proteomes (Mullaney et al., 2009; Olofsson et al., 2010; Zavan et al., 2019), there were also unique proteins ascribed to each one. Such differences in OMVs protein cargo might be related to the underlying growth conditions (bacterial strain, culture media, and initial bacterial density), which influence bacterial growth and the vesiculation process, in addition to technical issues (isolation method and proteomic analysis).

Considering the therapeutic potential of bacterial OMVs, it is of uttermost importance that the harvest of OMVs occurs from a chemically defined bacterial culture without animalderived components that hinder the purification process and ultimately interfere with the host immune response (van der Pol et al., 2015). Our method overcomes this issue and, by having increased time-efficiency in comparison to previous protocols, is a suitable approach for application in the biomedical field. Still, the yield and purity of $H$. pylori OMVs on a large-scale implementation of the protocol, and its application for the isolation of OMVs from other bacteria species, needs further evaluation. Currently, OMVs have been licensed for human use as adjuvant (Nokleby et al., 2007) or vaccine against the serogroup B Neisseria meningitides, namely the Bexsero ${ }^{\circledR}$ (Novartis) and VA-MENGOC-BC ${ }^{\circledR}$ (Finlay Institute, Cuba) vaccines (PetousisHarris, 2018), and other OMVs-based vaccines against infectious diseases are already in preclinical studies (Zhang et al., 2019). In the case of $H$. pylori, the development of an effective prophylactic or therapeutic vaccine, predominantly composed of purified or recombinant components of $H$. pylori antigens with an adjuvant, has proven challenging and not yet a reality (Sutton and Boag, 2019). Promising data showed that intragastric immunization with OMVs from $H$. pylori 7.13, resulted in the development of a specific systemic immune response in mice, and in the significant reduction of bacterial load after challenging with $H$. pylori SS1 (Liu et al., 2019; Song et al., 2020).

In conclusion, we described an abridged protocol for isolation of OMVs resorting to a synthetic chemically defined liquid medium for bacterial growth ensuing a high yield of pure and bona fide H. pylori OMVs, in a time- and cost-efficient manner, suitable for research, biomedical, and biopharmaceutical downstream applications.

\section{DATA AVAILABILITY STATEMENT}

The mass spectrometry proteomic data presented in the study is deposited in the ProteomeXchange Consortium via the PRIDE

\section{REFERENCES}

Adams, B. L., Bates, T. C., and Oliver, J. D. (2003). Survival of Helicobacter pylori in a natural freshwater environment. Appl. Environ. Microbiol. 69, 7462-7466. doi: $10.1128 /$ aem.69.12.7462-7466.2003 partner repository with the dataset identifier PXD025393 and doi: 10.6019/PXD025393.

\section{AUTHOR CONTRIBUTIONS}

JM and ML conceptualized the study. JM, VP, TF, AM, and HO acquired the data. JM, VP, TF, AM, HO, CF, and ML performed the data analysis and interpretation. JM drafted the manuscript. All authors revised the manuscript for important intellectual content. CF acquired the funding.

\section{FUNDING}

This article is a result of the project NORTE-01-0145-FEDER000029, supported by Norte Portugal Regional Program (NORTE 2020), under the PORTUGAL 2020 Partnership Agreement, through the European Regional Development Fund (ERDF). The i3S HEMS Scientific Platform is member of the national infrastructure PPBI - Portuguese Platform of Bioimaging (PPBIPOCI-01-0145-FEDER-022122). The i3S Proteomics Scientific Platform is funded by the Portuguese Mass Spectrometry Network, integrated in the National Roadmap of Research Infrastructures of Strategic Relevance (ROTEIRO/0028/2013; LISBOA-01-0145-FEDER-022125). JM and ML had fellowships from FCT - Fundação para a Ciência e a Tecnologia (SFRH/BD/116965/2016 and SFRH/BDP/110065/2015). ML has a FCT RJEC Id 3762 contract.

\section{ACKNOWLEDGMENTS}

The authors acknowledge the help of Bruno Cavadas for assistance in proteomic analysis, Cecília Durães for NTA acquisition, Rui Fernandes and Francisco Figueiredo for the contribution on the ultrastructural analysis of OMVs, Daniela Silva (CEMUP - Centro de Materiais da Universidade do Porto) for SEM acquisition, and Steeve Lima and Paulo Oliveira for sharing their optimized protocol of OMVs protein quantification and for the helpful discussions. The authors also thank the i3S HEMS and Proteomics Scientific Platforms.

\section{SUPPLEMENTARY MATERIAL}

The Supplementary Material for this article can be found online at: https://www.frontiersin.org/articles/10.3389/fmicb. 2021.654193/full\#supplementary-material

Atherton, J. C. (2006). The pathogenesis of Helicobacter pylori-induced gastroduodenal diseases. Annu. Rev. Pathol. 1, 63-96. doi: 10.1146/annurev.pathol. 1.110304 .100125

Azevedo, N. F., Almeida, C., Cerqueira, L., Dias, S., Keevil, C. W., and Vieira, M. J. (2007). Coccoid form of Helicobacter pylori as a morphological manifestation 
of cell adaptation to the environment. Appl. Environ. Microbiol. 73, 3423-3427. doi: 10.1128/AEM.00047-07

Bitto, N. J., and Kaparakis-Liaskos, M. (2017). The therapeutic benefit of bacterial membrane vesicles. Int. J. Mol. Sci. 18:1287. doi: 10.3390/ijms18061287

Blaser, M. J., and Atherton, J. C. (2004). Helicobacter pylori persistence: biology and disease. J. Clin. Invest. 113, 321-333. doi: 10.1172/JCI20925

Chatterjee, S. N., and Das, J. (1967). Electron microscopic observations on the excretion of cell-wall material by Vibrio cholerae. J. Gen. Microbiol. 49, 1-11. doi: 10.1099/00221287-49-1-1

Chitcholtan, K., Hampton, M. B., and Keenan, J. I. (2008). Outer membrane vesicles enhance the carcinogenic potential of Helicobacter pylori. Carcinogenesis 29, 2400-2405. doi: 10.1093/carcin/bgn218

Chutkan, H., Macdonald, I., Manning, A., and Kuehn, M. J. (2013). Quantitative and qualitative preparations of bacterial outer membrane vesicles. Methods Mol. Biol. 966, 259-272. doi: 10.1007/978-1-62703-245-2_16

Cover, T. L., and Blaser, M. J. (2009). Helicobacter pylori in health and disease. Gastroenterology 136, 1863-1873. doi: 10.1053/j.gastro.2009.01.073

de Bernard, M., and D'Elios, M. M. (2010). The immune modulating activity of the Helicobacter pylori HP-NAP: friend or foe? Toxicon 56, 1186-1192. doi: 10.1016/j.toxicon.2009.09.020

Deatherage, B. L., and Cookson, B. T. (2012). Membrane vesicle release in bacteria, eukaryotes, and archaea: a conserved yet underappreciated aspect of microbial life. Infect Immun. 80, 1948-1957. doi: 10.1128/IAI.06014- 11

Devoe, I. W., and Gilchrist, J. E. (1973). Release of endotoxin in the form of cell wall blebs during in vitro growth of Neisseria meningitidis. J. Exp. Med. 138, 1156-1167. doi: 10.1084/jem.138.5.1156

Fiocca, R., Necchi, V., Sommi, P., Ricci, V., Telford, J., Cover, T. L., et al. (1999). Release of Helicobacter pylori vacuolating cytotoxin by both a specific secretion pathway and budding of outer membrane vesicles. Uptake of released toxin and vesicles by gastric epithelium. J. Pathol. 188, 220-226.

Ge, R., and Sun, X. (2012). Iron trafficking system in Helicobacter pylori. Biometals 25, 247-258. doi: 10.1007/s10534-011-9512-8

Gonzalez-Lopez, M. A., and Olivares-Trejo, J. J. (2009). The gene frpB2 of Helicobacter pylori encodes an hemoglobin-binding protein involved in iron acquisition. Biometals 22, 889-894. doi: 10.1007/s10534-009-9240-5

Heczko, U., Smith, V. C., Mark Meloche, R., Buchan, A. M., and Finlay, B. B. (2000). Characteristics of Helicobacter pylori attachment to human primary antral epithelial cells. Microb. Infect 2, 1669-1676. doi: 10.1016/s1286-4579(00) 01322-8

Hoekstra, D., van der Laan, J. W., de Leij, L., and Witholt, B. (1976). Release of outer membrane fragments from normally growing Escherichia coli. Biochim. Biophys. Acta 455, 889-899. doi: 10.1016/0005-2736(76)90058-4

Hooi, J. K. Y., Lai, W. Y., Ng, W. K., Suen, M. M. Y., Underwood, F. E., Tanyingoh, D., et al. (2017). Global prevalence of Helicobacter pylori infection: systematic review and meta-analysis. Gastroenterology 153, 420-429. doi: 10.1053/j.gastro. 2017.04.022

Hughes, C. S., Moggridge, S., Muller, T., Sorensen, P. H., Morin, G. B., and Krijgsveld, J. (2019). Single-pot, solid-phase-enhanced sample preparation for proteomics experiments. Nat. Protoc. 14, 68-85. doi: 10.1038/s41596-018-0 082-x

Hutton, M. L., Kaparakis-Liaskos, M., Turner, L., Cardona, A., Kwok, T., and Ferrero, R. L. (2010). Helicobacter pylori exploits cholesterolrich microdomains for induction of NF-kappaB-dependent responses and peptidoglycan delivery in epithelial cells. Infect Immun. 78, 4523-4531. doi: 10.1128/IAI.00439-10

Ierardi, E., Losurdo, G., Mileti, A., Paolillo, R., Giorgio, F., Principi, M., et al. (2020). The puzzle of coccoid forms of Helicobacter pylori: beyond basic science. Antibiotics 9:293. doi: 10.3390/antibiotics9060293

Ishijima, N., Suzuki, M., Ashida, H., Ichikawa, Y., Kanegae, Y., Saito, I., et al. (2011). BabA-mediated adherence is a potentiator of the Helicobacter pylori type IV secretion system activity. J. Biol. Chem. 286, 25256-25264. doi: 10.1074/jbc. M111.233601

Ismail, S., Hampton, M. B., and Keenan, J. I. (2003). Helicobacter pylori outer membrane vesicles modulate proliferation and interleukin- 8 production by gastric epithelial cells. Infect Immun. 71, 5670-5675. doi: 10.1128/iai.71.10. 5670-5675.2003

Jarzab, M., Posselt, G., Meisner-Kober, N., and Wessler, S. (2020). Helicobacter pylori-derived outer membrane vesicles (OMVs): role in bacterial pathogenesis? Microorganisms 8:1328. doi: 10.3390/microorganisms8091328
Jimenez-Soto, L. F., Rohrer, S., Jain, U., Ertl, C., Sewald, X., and Haas, R. (2012). Effects of cholesterol on Helicobacter pylori growth and virulence properties in vitro. Helicobacter 17, 133-139. doi: 10.1111/j.1523-5378.2011.0 0926.x

Kaparakis, M., Turnbull, L., Carneiro, L., Firth, S., Coleman, H. A., Parkington, H. C., et al. (2010). Bacterial membrane vesicles deliver peptidoglycan to NOD1 in epithelial cells. Cell Microbiol. 12, 372-385. doi: 10.1111/j.1462-5822.2009. 01404.x

Katsui, N., Tsuchido, T., Hiramatsu, R., Fujikawa, S., Takano, M., and Shibasaki, I. (1982). Heat-induced blebbing and vesiculation of the outer membrane of Escherichia coli. J. Bacteriol. 151, 1523-1531. doi: 10.1128/JB.151.3.1523-1531. 1982

Keenan, J., Day, T., Neal, S., Cook, B., Perez-Perez, G., Allardyce, R., et al. (2000). A role for the bacterial outer membrane in the pathogenesis of Helicobacter pylori infection. FEMS Microbiol. Lett. 182, 259-264. doi: 10.1111/j.1574-6968.2000. tb08905.x

Kim, G. H., Choi, C. W., Park, E. C., Lee, S. Y., and Kim, S. I. (2014). Isolation and proteomic characterization of bacterial extracellular membrane vesicles. Curr. Protein Pept. Sci. 15, 719-731. doi: 10.2174/1573403x10666140505163121

Klimentova, J., and Stulik, J. (2015). Methods of isolation and purification of outer membrane vesicles from gram-negative bacteria. Microbiol. Res. 170, 1-9. doi: 10.1016/j.micres.2014.09.006

Kuhns, L. G., Wang, G., and Maier, R. J. (2015). Comparative roles of the two Helicobacter pylori thioredoxins in preventing macromolecule damage. Infect Immun. 83, 2935-2943. doi: 10.1128/IAI.00232-15

Kulp, A., and Kuehn, M. J. (2010). Biological functions and biogenesis of secreted bacterial outer membrane vesicles. Annu. Rev. Microbiol. 64, 163-184. doi: 10.1146/annurev.micro.091208.073413

Kusters, J. G., Gerrits, M. M., Van Strijp, J. A., and Vandenbroucke-Grauls, C. M. (1997). Coccoid forms of Helicobacter pylori are the morphologic manifestation of cell death. Infect Immun. 65, 3672-3679. doi: 10.1128/IAI.65.9.3672-3679. 1997

Liu, Q., Li, X., Zhang, Y., Song, Z., Li, R., Ruan, H., et al. (2019). Orally-administered outer-membrane vesicles from Helicobacter pylori reduce H. pylori infection via Th2-biased immune responses in mice. Pathog. Dis. 77:ftz050. doi: 10.1093/femspd/ftz050

Marshall, B. J., Barrett, L. J., Prakash, C., McCallum, R. W., and Guerrant, R. L. (1990). Urea protects Helicobacter (Campylobacter) pylori from the bactericidal effect of acid. Gastroenterology 99, 697-702. doi: 10.1016/0016-5085(90)90 957-3

Mullaney, E., Brown, P. A., Smith, S. M., Botting, C. H., Yamaoka, Y. Y., Terres, A. M., et al. (2009). Proteomic and functional characterization of the outer membrane vesicles from the gastric pathogen Helicobacter pylori. Proteom. Clin. Appl. 3, 785-796. doi: 10.1002/prca.200800192

Mytilinaios, I., Salih, M., Schofield, H. K., and Lambert, R. J. (2012). Growth curve prediction from optical density data. Int. J. Food Microbiol. 154, 169-176. doi: 10.1016/j.ijfoodmicro.2011.12.035

Nokleby, H., Aavitsland, P., O’Hallahan, J., Feiring, B., Tilman, S., and Oster, P. (2007). Safety review: two outer membrane vesicle (OMV) vaccines against systemic Neisseria meningitidis serogroup B disease. Vaccine 25, 3080-3084. doi: 10.1016/j.vaccine.2007.01.022

Odenbreit, S., Wieland, B., and Haas, R. (1996). Cloning and genetic characterization of Helicobacter pylori catalase and construction of a catalasedeficient mutant strain. J. Bacteriol. 178, 6960-6967. doi: 10.1128/jb.178.23. 6960-6967.1996

Olofsson, A., Vallstrom, A., Petzold, K., Tegtmeyer, N., Schleucher, J., Carlsson, S., et al. (2010). Biochemical and functional characterization of Helicobacter pylori vesicles. Mol. Microbiol. 77, 1539-1555. doi: 10.1111/j.1365-2958.2010.07 307.x

Parker, H., Chitcholtan, K., Hampton, M. B., and Keenan, J. I. (2010). Uptake of Helicobacter pylori outer membrane vesicles by gastric epithelial cells. Infect Immun. 78, 5054-5061. doi: 10.1128/IAI.00299-10

Perez-Riverol, Y., Csordas, A., Bai, J., Bernal-Llinares, M., Hewapathirana, S., Kundu, D. J., et al. (2019). The PRIDE database and related tools and resources in 2019: improving support for quantification data. Nucleic Acids Res. 47, D442-D450. doi: 10.1093/nar/gky1106

Pesci, E. C., and Pickett, C. L. (1994). Genetic organization and enzymatic activity of a superoxide dismutase from the microaerophilic human pathogen, Helicobacter pylori. Gene 143, 111-116. doi: 10.1016/0378-1119(94)90614-9 
Petousis-Harris, H. (2018). Impact of meningococcal group B OMV vaccines, beyond their brief. Hum. Vaccin. Immunother. 14, 1058-1063. doi: 10.1080/ 21645515.2017.1381810

Ricci, V., Sommi, P., Fiocca, R., Romano, M., Solcia, E., and Ventura, U. (1997). Helicobacter pylori vacuolating toxin accumulates within the endosomalvacuolar compartment of cultured gastric cells and potentiates the vacuolating activity of ammonia. J. Pathol. 183, 453-459. doi: 10.1002/(SICI)10969896(199712)183:4<453:AID-PATH950<3.0.CO;2-2

Schoep, T. D., Fulurija, A., Good, F., Lu, W., Himbeck, R. P., Schwan, C., et al. (2010). Surface properties of Helicobacter pylori urease complex are essential for persistence. PLoS One 5:e15042. doi: 10.1371/journal.pone.0 015042

Shibayama, K., Kamachi, K., Nagata, N., Yagi, T., Nada, T., Doi, Y., et al. (2003). A novel apoptosis-inducing protein from Helicobacter pylori. Mol. Microbiol. 47, 443-451. doi: 10.1046/j.1365-2958.2003.03305.x

Song, Z., Li, B., Zhang, Y., Li, R., Ruan, H., Wu, J., et al. (2020). Outer membrane vesicles of Helicobacter pylori 7.13 as adjuvants promote protective efficacy against Helicobacter pylori infection. Front. Microbiol. 11:1340. doi: 10.3389/ fmicb. 2020.01340

Sutton, P., and Boag, J. M. (2019). Status of vaccine research and development for Helicobacter pylori. Vaccine 37, 7295-7299. doi: 10.1016/j.vaccine.2018.01.001

Tegtmeyer, N., Wessler, S., Necchi, V., Rohde, M., Harrer, A., Rau, T. T., et al. (2017). Helicobacter pylori employs a unique basolateral Type IV secretion mechanism for CagA delivery. Cell Host Microb. 22, 552-560.e555. doi: 10.1016/ j.chom.2017.09.005

Testerman, T. L., Conn, P. B., Mobley, H. L., and McGee, D. J. (2006). Nutritional requirements and antibiotic resistance patterns of Helicobacter species in chemically defined media. J. Clin. Microbiol. 44, 1650-1658. doi: 10.1128/JCM. 44.5.1650-1658.2006

Testerman, T. L., McGee, D. J., and Mobley, H. L. (2001). Helicobacter pylori growth and urease detection in the chemically defined medium Ham's F-12 nutrient mixture. J. Clin. Microbiol. 39, 3842-3850. doi: 10.1128/JCM.39.11. 3842-3850.2001

Teymournejad, O., Mobarez, A. M., Hassan, Z. M., and Talebi Bezmin Abadi, A. (2017). Binding of the Helicobacter pylori OipA causes apoptosis of host cells via modulation of Bax/Bcl-2 levels. Sci. Rep. 7:8036. doi: 10.1038/s41598-01708176-7

Thompson, S. S., Naidu, Y. M., and Pestka, J. J. (1985). Ultrastructural localization of an extracellular protease in Pseudomonas fragi by using the peroxidaseantiperoxidase reaction. Appl. Environ. Microbiol. 50, 1038-1042. doi: 10.1128/ AEM.50.4.1038-1042.1985
Tseng, Y. S., Wu, D. C., Chang, C. Y., Kuo, C. H., Yang, Y. C., Jan, C. M., et al. (2009). Amoxicillin resistance with beta-lactamase production in Helicobacter pylori. Eur. J. Clin. Invest. 39, 807-812. doi: 10.1111/j.1365-2362.2009.02166.x

Tsolakos, N., Lie, K., Bolstad, K., Maslen, S., Kristiansen, P. A., Hoiby, E. A., et al. (2010). Characterization of meningococcal serogroup B outer membrane vesicle vaccines from strain 44/76 after growth in different media. Vaccine 28, 3211-3218. doi: 10.1016/j.vaccine.2010.02.023

Turner, L., Bitto, N. J., Steer, D. L., Lo, C., D’Costa, K., Ramm, G., et al. (2018). Helicobacter pylori outer membrane vesicle size determines their mechanisms of host cell entry and protein content. Front. Immunol. 9:1466. doi: 10.3389/ fimmu.2018.01466

van der Pol, L., Stork, M., and van der Ley, P. (2015). Outer membrane vesicles as platform vaccine technology. Biotechnol. J. 10, 1689-1706. doi: 10.1002/biot. 201400395

Willen, R., Carlen, B., Wang, X., Papadogiannakis, N., Odselius, R., and Wadstrom, T. (2000). Morphologic conversion of Helicobacter pylori from spiral to coccoid form. Scanning (SEM) and transmission electron microscopy (TEM) suggest viability. Ups. J. Med. Sci. 105, 31-40. doi: 10.1517/03009734000000045

Yamaoka, Y., Kikuchi, S., El-Zimaity, H. M., Gutierrez, O., Osato, M. S., and Graham, D. Y. (2002). Importance of Helicobacter pylori oipA in clinical presentation, gastric inflammation, and mucosal interleukin 8 production. Gastroenterology 123, 414-424. doi: 10.1053/gast.2002.34781

Zavan, L., Bitto, N. J., Johnston, E. L., Greening, D. W., and Kaparakis-Liaskos, M. (2019). Helicobacter pylori growth stage determines the size, protein composition, and preferential cargo packaging of outer membrane vesicles. Proteomics 19:e1800209. doi: 10.1002/pmic.201800209

Zhang, Y., Fang, Z., Li, R., Huang, X., and Liu, Q. (2019). Design of outer membrane vesicles as cancer vaccines: a new toolkit for cancer therapy. Cancers 11:1314. doi: 10.3390/cancers 11091314

Conflict of Interest: The authors declare that the research was conducted in the absence of any commercial or financial relationships that could be construed as a potential conflict of interest.

Copyright (c) 2021 Melo, Pinto, Fernandes, Malheiro, Osório, Figueiredo and Leite. This is an open-access article distributed under the terms of the Creative Commons Attribution License (CC BY). The use, distribution or reproduction in other forums is permitted, provided the original author(s) and the copyright owner(s) are credited and that the original publication in this journal is cited, in accordance with accepted academic practice. No use, distribution or reproduction is permitted which does not comply with these terms. 